\title{
Bilateral blindness with secondary retinitis pigmentosa following postoperative docetaxel and platinum combination chemotherapy in primary small-cell carcinoma of the endometrium: An unusual case report and review of the literature
}

\author{
QIONG-LAN TANG ${ }^{1 *}$, JIA LIU $^{2 *}, \mathrm{LI} \mathrm{ZUO}^{2 *}, \mathrm{CONG} \mathrm{CHI}^{2}$, \\ HUI-YU DONG ${ }^{2}$, XIAO-XIA JIANG ${ }^{2}$ and XUE-FENG JIANG ${ }^{2}$ \\ ${ }^{1}$ Department of Pathology, Sun Yat-Sen Memorial Hospital, Sun Yat-Sen University, Guangzhou, Guangdong 510120; \\ ${ }^{2}$ Department of Obstetrics and Gynecology, The First Affiliated Hospital, \\ Jinan University, Guangzhou, Guangdong 510630, P.R. China
}

Received October 13, 2016; Accepted January 10, 2017

DOI: $10.3892 / \mathrm{mco} .2017 .1174$

\begin{abstract}
Ocular toxicity is an uncommon complication of cytotoxic chemotherapy. Bilateral blindness with secondary retinitis pigmentosa (RP) following docetaxel and platinum combination chemotherapy at the recommended dose is extremely rare. The present study reports a case of advanced small-cell carcinoma (SCC) of the endometrium in a patient with diabetes mellitus type 2 . The patient suffered from RP with a sharp decline in vision after the fourth course of postoperative docetaxel and platinum combination chemotherapy. Unfortunately, the patient developed bilateral blindness after another course of chemotherapy at a reduced dose. No tumor recurrence was observed during the 33 months of follow-up. A total of 35 cases of docetaxel- and/or platinum-induced retinal toxicity were found in the English literature and reviewed. The ischemic and electrophysiological hypotheses may have been implicated in the pathogenesis of ocular toxicity in the present case, particularly with the history of diabetes. Understanding the ocular side effects of this combination chemotherapy may assist gynecological oncologists and ophthalmologists with early recognition and timely intervention before blindness is established.
\end{abstract}

Correspondence to: Dr Xue-Feng Jiang, Department of Obstetrics and Gynecology, The First Affiliated Hospital, Jinan University, 613 Huangpu West Road, Guangzhou, Guangdong 510630, P.R. China E-mail: xuefengjiang@126.com

*Contributed equally

Key words: small-cell carcinoma, endometrium, secondary retinitis pigmentosa, bilateral blindness, docetaxel, platinum

\section{Introduction}

Ocular toxicity induced by cancer chemotherapy includes a broad spectrum of disorders, such as cortical blindness, blurred vision and maculopathy, reflecting the unique anatomical, physiological and biochemical characteristics of the eye. The ocular side effects may be grouped into adnexal, anterior segment, posterior segment and neuro-ophthalmic. Posterior segment lesions are important due to the marked visual loss that may occur (1). Visual loss secondary to secondary retinitis pigmentosa (RP) is an uncommon complication of cytotoxic chemotherapy. Docetaxel and platinum have been frequently used in combination to treat various solid tumors, and are relatively rarely associated with severe side effects (2). Although secondary RP with bilateral blindness following docetaxel and platinum combination chemotherapy is extremely rare, this complication warrants attention due to its devastating impact on the quality of life of patients who receive various anticancer therapies. We herein report a case of a patient with small-cell carcinoma (SCC) of the endometrium who suffered from bilateral blindness during postoperative chemotherapy at the recommended dose, and review docetaxel- and/or platinum-induced retinal toxicity in terms of clinical manifestations, diagnostic and preventive strategies, with the aim of improving patient safety during anticancer therapy.

\section{Case report}

On 13th October 2013, a 48-year-old Chinese woman was admitted to the Department of Obstetrics and Gynecology of the First Affiliated Hospital of Jinan University (Guangzhou, China) complaining of irregular vaginal bleeding for 1 month. Magnetic resonance imaging (MRI) revealed a $5.0-\mathrm{cm}$ mass in the endometrial cavity and cervical stromal invasion. Dilation and curettage and histological examination revealed SCC of the endometrium involving the cervix. Immunohistochemical examination showed positive staining for synaptophysin, chromogranin A and neuron-specific enolase in the tumor cells. 


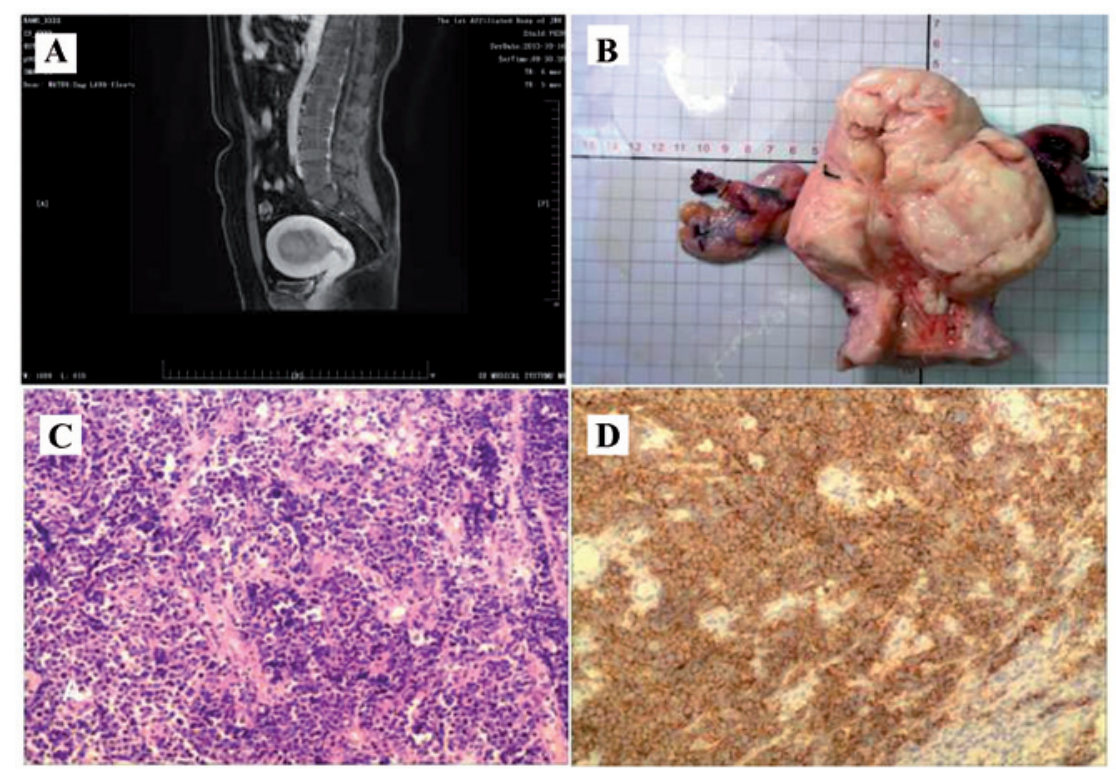

Figure 1. Morphological changes of small-cell carcinoma (SCC) of the endometrium. (A) Sagittal enhanced magnetic resonance imaging showing the endometrial lesion. (B) Macroscopic specimen after the operation showing the tumor of the endometrium. (C) Hematoxylin and eosin staining showing SCC of the endometrium (magnification, x200). (D) Positive immunohistochemical staining for synaptophysin (magnification, x200).
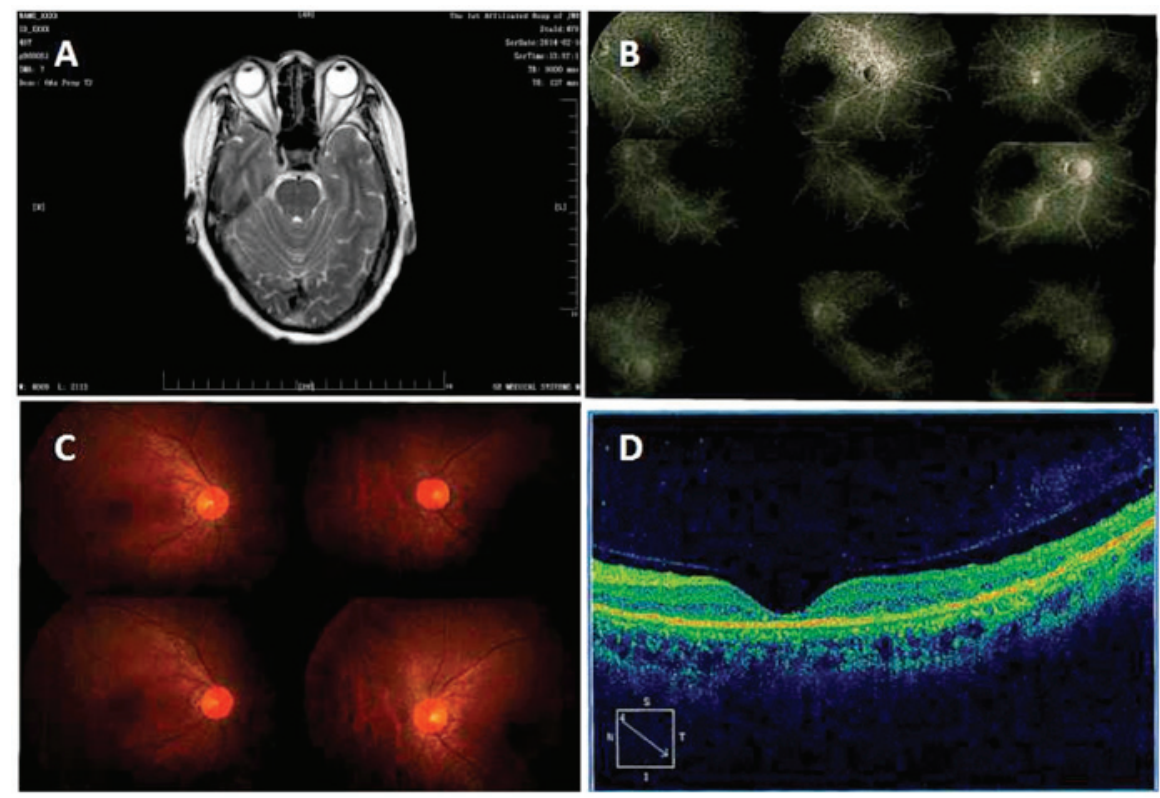

Figure 2. Imaging of the eye in a patient with bilateral blindness. (A) No metastatic lesions were found in the bilateral orbital and optic nerves on axial T2-weighted magnetic resonance imaging. (B) Fundus fluorescence angiography showing depigmentation with macular dark areas. (C) Fundus photograph showing secondary retinal pigment degeneration. (D) Optical coherence tomography showing thinning of the macular retina.

The patient also had a 1-year history of diabetes mellitus type 2 , treated with $50 \mathrm{mg}$ acarbose per day. The blood pressure and blood sugar levels were normal, there were no visual abnormalities, and other systemic investigations were also negative. After systemic metastasis of SCC was excluded, the patient underwent open extensive hysterectomy, bilateral salpingo-oophorectomy and pelvic lymph node dissection. Pathological evaluation revealed that $\sim 90 \%$ of the tumor in the corpus uteri was SCC with myometrial invasion and cervical involvement, and $10 \%$ was endometrioid adenocarcinoma (Fig. 1). Metastatic SCC was also observed in the left external iliac and obturator lymph nodes. The patient was diagnosed with SCC of the endometrium, International Federation of Gynecology and Obstetrics stage IIIC1 (3). Docetaxel and platinum combination adjuvant chemotherapy was administered after the operation. After the first four courses of chemotherapy with docetaxel $120 \mathrm{mg}\left(70 \mathrm{mg} / \mathrm{m}^{2}\right)$ and cisplatin $90 \mathrm{mg}\left(50 \mathrm{mg} / \mathrm{m}^{2}\right)$ at 3 -week intervals, the patient complained of visual impairment, particularly farsightedness. The visual acuities were 0.3 on the right and 0.5 on the left side, and MRI revealed no intracranial space-occupying lesion. Following exclusion of ophthalmological contraindications, the fifth 


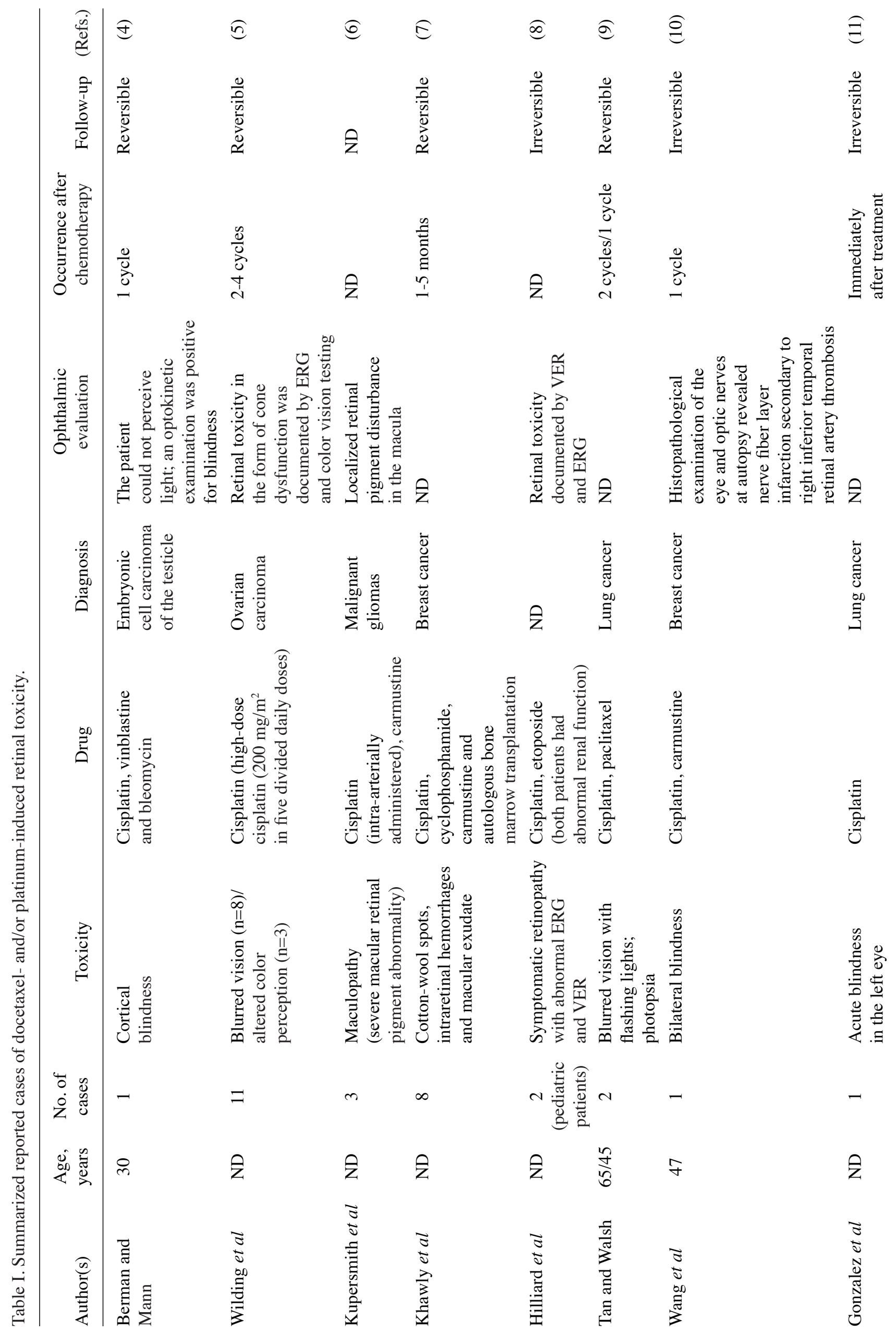




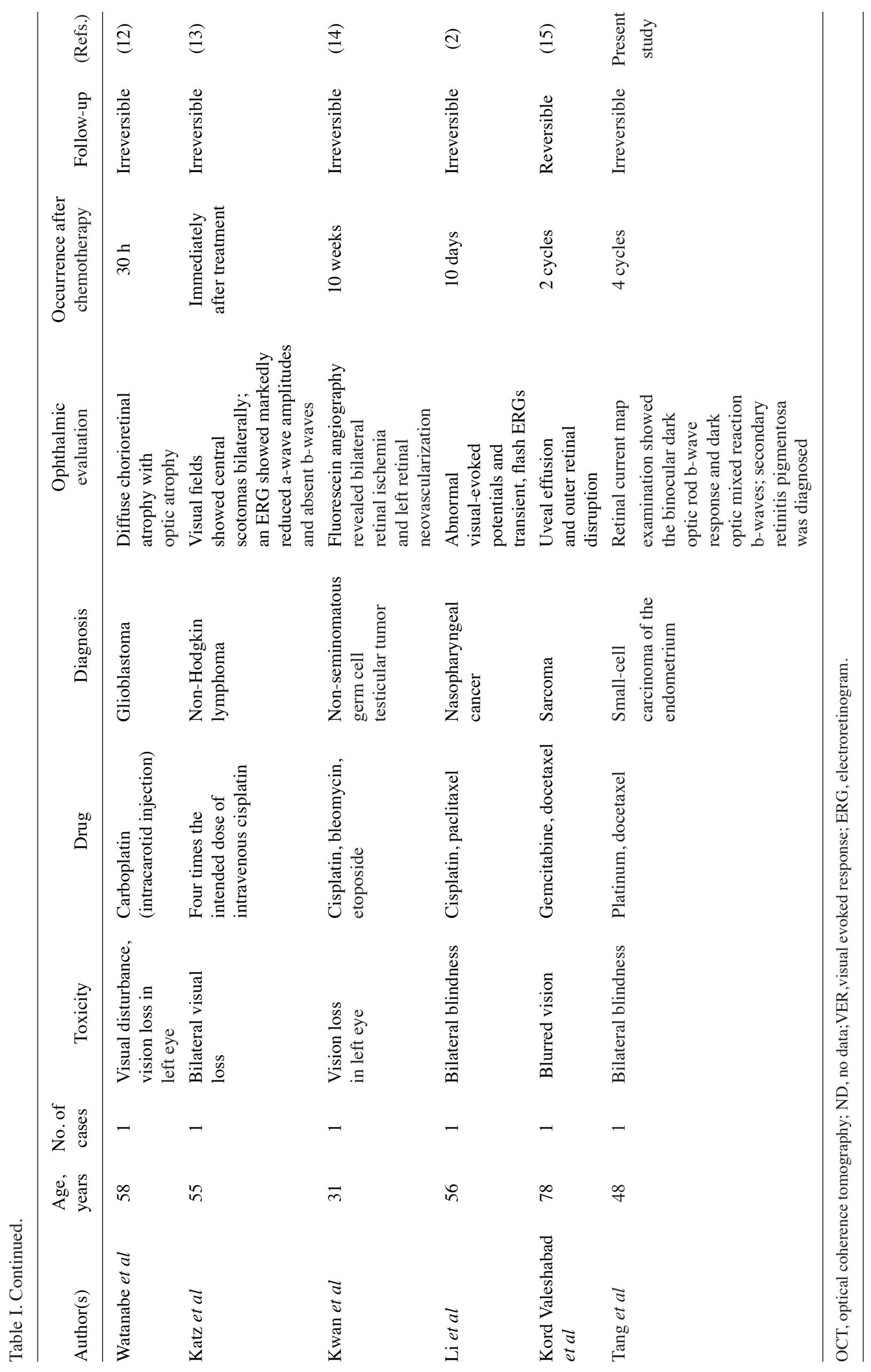


course of chemotherapy consisted of docetaxel $120 \mathrm{mg}$ $\left(70 \mathrm{mg} / \mathrm{m}^{2}\right)$ and reduced lobaplatin $45 \mathrm{mg}\left(25 \mathrm{mg} / \mathrm{m}^{2}\right)$ was administered. After 2 weeks, the patient was admitted to the hospital for worsening vision with light perception. Fundus fluorescence angiography revealed early-stage diffuse depigmentation of the retinal pigment epithelium and a window defect (hyperfluorescence) involving the macular area in both eyes. Electroretinography revealed loss of function of rod and cone cells bilaterally. Optical coherence tomography showed thinning of the macular retina (Fig. 2). Retinal current map examination showed that the binocular dark optic rod b-wave response and dark optic mixed reaction b-waves were not recorded, and absence of photopic a- and b-wave cone response. Fundus angiography showed secondary RP. Treatments aimed at improving fundus microcirculation were performed, including administration of enteric-coated aspirin and tanshinone. Unfortunately, the visual acuity of the patient did not improve following cessation of chemotherapy. There was no tumor recurrence during the 33-month follow-up, but the patient developed bilateral blindness.

The patient provided written informed consent to the publication of this case report and associated images.

\section{Discussion}

Although postoperative adjuvant chemotherapy with docetaxel and platinum is an increasingly used regimen for advanced/ metastatic or recurrent endometrial cancer with lower toxicity and good tolerability (3), some of these toxicities are irreversible and may adversely affect the patient's quality of life, or even cause a permanent disability (2). To the best of our knowledge, RP with bilateral blindness following docetaxel and platinum combination chemotherapy has not yet been reported in the English literature.

Of note, paraneoplastic retinopathy-related bilateral blindness from SCC of the endometrium should be excluded. The initial symptoms of paraneoplastic retinopathy-related bilateral blindness were decreased visual acuity and narrowing of the visual field, or blindness on exposure to bright light and total achromatopsia. In the present case, the patient first presented with visual disturbances $>2$ months after the diagnosis of SCC of the endometrium. Visual disturbances associated with malignant neoplasms are more often caused by metastasis to the brain, meninges, optic nerve, orbit, choroid, or retina. MRI revealed no intracranial space-occupying lesion and metastatic brain tumor from SCC of the endometrium was also excluded.

A total of 35 cases involving docetaxel- and/or platinum-related retinal toxicity have been reported and are summarized in Table I. The mean age of the patients was 51.3 years. Vision loss developed immediately after treatment in 2 cases, whereas in the remaining cases it developed after 10 days to 4 cycles of chemotherapy. A total of 7 cases suffered from bilateral blindness or hemianopia. A total of 24 cases were reversible and 8 cases were irreversible at follow-up. Retinal toxicity was more severe in 4 patients who received intra-arterially administered cisplatin chemotherapy for brain malignancy.

Retinal toxicity includes maculopathy in the form of pigmentary changes attributable to localized retinal pigment disturbance, altered color perception attributable to cone dysfunction, and mild retinal ischemic changes, such as cotton-wool spots and intraretinal hemorrhages in the posterior pole (14).

The mechanism underlying ocular neurotoxicity remains unknown and the ischemic and electrophysiological hypotheses may be involved in the pathogenesis of ocular toxicity. Cisplatin-associated neurotoxicity is dose-dependent. Severe neurotoxicity is extremely uncommon at a cumulative dose $<400 \mathrm{mg} / \mathrm{m}^{2}$, but the incidence increases at a cumulative dose of $600-800 \mathrm{mg} / \mathrm{m}^{2}$ (2).Occlusion of a retinal artery branch, severe macular ischemia or retinal neovascularization are associated with high-dose platinum. Cisplatin increases human platelet reactivity (onset of platelet aggregation wave and thromboxane production) to non-aggregating concentrations of the agonists involving arachidonic acid metabolism (16). In addition, a study on autopsy specimens also identified focal small-vessel thrombosis and vessel occlusion as the cause of blindness in a patient on high-dose carmustine and cisplatin therapy (10).

The visual symptoms and electrophysiological changes following intravenous paclitaxel administration were likely caused by retinal vascular dysregulation or optic nerve ischemia (17). As the cystoid macular edema occurred following treatment with paclitaxel, one possible theory is that Müller cell toxicity results from intracellular fluid accumulation and subclinical extracellular fluid leakage. Reversible uveal effusion and outer retinal disruption were reported following gemcitabine and docetaxel chemotherapy (15).

Bakbak et al (18) reported that systemic administration of cisplatin and paclitaxel affected the peripapillary retinal nerve fibre layer thickness and visual field index, as revealed by frequency-doubling technology (FDT) perimetry. Optical coherence tomography and FDT perimetry may be adjunctive tools for the screening of ocular toxicity in patients treated with these agents.

It has been reported that children and those patients with renal dysfunc-tion or diabetes mellitus appear to be the highest risk groups for cisplatin-related neurotoxicity $(19,20)$. Patients with diabetic complications may opt to avoid paclitaxel plus platinum combination therapies if there are alternative effective treatment options available (20). Unfortunately, our patient suffered from diabetes and developed an apparent accelerated decline in visual function during conventional adjuvant chemotherapy; an alternative regimen of adjuvant radiotherapy was not applied.

Due to its rarity, retinal toxicity may be underes-timated or considered as a minor complication when compared with other life-threatening complications. Understanding the ocular side effects of chemotherapy may assist ophthalmologists and oncologists with early identification and timely intervention, before blindness becomes established.

\section{References}

1. Omoti AE and Omoti CE: Ocular toxicity of systemic anticancer chemotherapy. Pharm Pract (Granada) 4: 55-59, 2006.

2. Li Y, Li Y, Li J, Pi G and Tan W: Paclitaxel- and/or cisplatin-induced ocular neurotoxicity: A case report and literature review. Onco Targets Ther 7: 1361-1366, 2014.

3. Uterine Neoplasms: NCCN Guidelines Version 2: MS-19, 2016.

4. Berman IJ and Mann MP: Seizures and transient cortical blindness associ-ated with cisplatinum (II) diamminedichloride (PDD) therapy in a thirty-year-old man. Cancer 45: 764-766, 1980. 
5. Wilding G, Caruso R, Lawrence TS, Ostchega Y, Ballintine EJ, Young RC and Ozols RF: Retinal toxicity after high dose cisplatin therapy. J Clin Oncol 3: 1683-1689, 1985.

6. Kupersmith MJ, Seiple WH, Holopigian K, Noble K, Hiesiger E and Warren F: Maculopathy caused by intra-arterially administered cisplatin and intravenously administered carmustine. Am J Ophthalmol 113: 435-438, 1992.

7. Khawly JA, Rubin P, Petros W, Peters WP and Jaffe GJ: Retinopathy and optic neuropathy in bone marrow transplantation for breast cancer. Ophthalmology 103: 87-95, 1996.

8. Hilliard LM, Berkow RL, Watterson J, Ballard EA, Balzer GK and Moertel CL: Retinal toxicity associated with cisplatin and etoposide in pediatric patients. Med Pediatr Oncol 28: 310-313, 1997.

9. Tan WW and Walsh T: Ocular toxicity secondary to paclitaxel in two lung cancer patients. Med Pediatr Oncol 31: 177, 1998.

10. Wang MY, Arnold AC, Vinters HV and Glasgow BJ: Bilateral blindness and lumbosacral myelopathy associated with high-dose carmustine and cisplatin therapy. Am J Ophthalmol 130: 367-368, 2000.

11. Gonzalez F, Menendez D and Gomez-Ulla F: Monocular visual loss in a patient undergoing cisplatin chemotherapy. Int Ophthalmol 24: 301-304, 2001.

12. Watanabe W, Kuwabara R, Nakahara T, Hamasaki O, Sakamoto I, Okada K, Minamoto A and Mishima HK: Severe ocular and orbital toxicity after intracarotid injection of carboplatin for recurrent glioblastomas. Graefes Arch Clin Exp Ophthalmol 240: $1033-1035,2002$.
13. Katz BJ, Ward JH, Digre KB, Creel DJ and Mamalis N: Persistent severe visual and electroretinographic abnormalities after intravenous Cisplatin therapy. J Neuroophthalmol 23: 132-135, 2003.

14. Kwan AS, Sahu A and Palexes G: Retinal ischemia with neovascularization in cisplatin related retinal toxicity. Am J Ophthalmol 141: 196-197, 2006.

15. Kord Valeshabad A, Mieler WF, Setlur V, Thomas M and Shahidi M: Posterior segment toxicity after gemcitabine and docetaxel chemotherapy. Optom Vis Sci 92: e110-e113, 2015.

16. Togna GI, Togna AR, Franconi M and Caprino L: Cisplatin triggers platelet activation. Thromb Res 99: 503-509, 2000.

17. Scaioli V, Caraceni A, Martini C, Curzi S, Capri G and Luca G: Electrophysiological evaluation of visual pathways in paclitaxel-treated patients. J Neurooncol 77: 79-87, 2006.

18. Bakbak B, Gedik S, Koktekir BE, Yavuzer K, Tulek B, Kanat F and Pancar E: Assessment of ocular neurotoxicity in patients treated with systemic cancer chemotherapeutics. Cutan Ocul Toxicol 33: 7-10, 2014.

19. Walsh TJ, Clark AW, Parhad IM and Green WR: Neurotoxic effects of cisplatin therapy. Arch Neurol 39: 719-720, 1982.

20. Hershman DL, Till C, Wright JD, Awad D, Ramsey SD, Barlow WE, Minasian LM and Unger J: Comorbidities and risk of chemotherapy-induced peripheral neuropathy among participants 65 years or older in southwest oncology group clinical trials. J Clin Oncol 34: 3014-3022, 2016. 